\title{
Student understanding of unit vectors and coordinate systems beyond cartesian coordinates in upper division physics courses
}

\author{
Marlene Vega ${ }^{1}$, Warren Christensen ${ }^{2}$, Brian Farlow ${ }^{2}$, Gina Passante ${ }^{1}$, Michael Loverude ${ }^{1}$ \\ Department of Physics, California State University Fullerton, Fullerton, CA 92834, USA \\ Department of Physics, North Dakota State University, Fargo, ND 58108, USA
}

In upper division physics courses students are required to work with various coordinate systems. This skill becomes particularly important when learning Electricity and Magnetism, where the most appropriate coordinate system will often depend on the geometry and symmetry of a problem. This study aims to identify and describe "resources" used by students when answering physics questions regarding unit vectors in non-Cartesian coordinate systems, specifically polar coordinates [1]. Data were collected in the form of written responses and interviews in upper division physics courses at two universities. After deeper analysis we identified several resources that students use in ways that can be productive and unproductive.

\section{INTRODUCTION}

This work is part of a collaborative study to investigate student learning of mathematics in the context of upperdivision physics courses, particularly the math methods courses offered in many physics departments. While PER has primarily focused on introductory-level courses, there are increasing efforts to expand into the upper division [2]. Upper division physics courses such as Mechanics and Electricity and Magnetism require a considerable understanding of challenging mathematical concepts. For example, the ability to incorporate the use of vectors in different coordinate systems is an essential skill in these courses. Many previous studies have shown that students continue to struggle with making sense of the mathematics necessary to solve physics problems [3].

In this portion of the project we have chosen to focus on a small portion of the mathematics used in upper division coursework: the unit vector formalism applied in the context of motion in two dimensions. Previous work has also focused on unit vectors in polar coordinates and spherical coordinates [4- 7]. For this paper, rather than simply looking at what students are doing right and wrong, we seek to identify and describe "resources" activated by students when answering physics questions regarding unit vectors polar coordinates.

\section{THEORETICAL FRAMEWORK}

This research project was originally guided by a framework consistent with 'identifying student difficulties.' Our goal was to study and describe student reasoning and to improve instruction by targeting student difficulties [8]. However, during our analysis of the written data, we came to realize that this framework was limiting when it came to explaining the various incorrect responses we were seeing, and did not take into account the productive resources that students may have been using when constructing their answer [8]. Student interviews provided additional motivation to consider a resource-based model. In these interviews, students' responses were not consistent with stable, strongly-held ideas; rather, student responses seemed to be more fluid and context dependent. In addition, incorrect answers involved elements of correct thinking, and a resource framework allowed us to make use of the productive resources that students access and build upon them, as well as to identify unproductively applied resources.

\section{RESEARCH METHODS}

\section{Written Responses:}

The original unit vector question (Figure 1) was part of a multipart problem given to physics majors $(N=49)$ at two different universities; California State University Fullerton (CSUF) and North Dakota State University (NDSU). While we decided to focus on this question, there are others who focused on other parts of the multipart problem [9]. At NDSU the question was given as an ungraded quiz in an E\&M Theory course before any instruction. For CSUF, this question was included on course examinations in the Mathematical Methods of Physics course ("Math Methods"). The Math Methods course was taught by a study author and PER professor who made this a topic a focus in their class.

口

Consider the motion of a particle in a plane as shown. For the locations shown, draw an arrow in the box to indicate the direction of the following vectors:
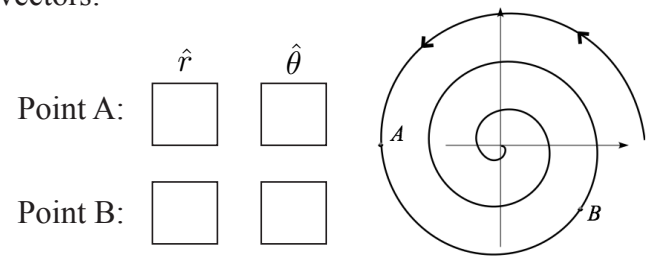

Figure 1. One of two versions of the original unit vector question given to students on an exam or pretest. The second version is identical but with the motion as shown in response (4) in Figure 3.

In order to be able to answer this question correctly, students need to know that $\hat{r}$ points in the direction of increasing $\mathrm{r}$ and $\hat{\theta}$ points in the direction of increasing $\theta$, 
by convention in a counter-clockwise direction starting from the positive $\mathrm{x}$-axis. As this is an orthonormal basis, $\hat{\theta}$ and $\hat{r}$ are orthogonal to one another. Lastly, for polar coordinates this basis is local; the directions will depend on the location of the point chosen. For points $\mathrm{A}$ and $\mathrm{B}, \hat{r}$ points away from the origin at each location and $\hat{\theta}$ is perpendicular to $\hat{r}$ in a counterclockwise direction. The written responses were coded and assigned to categories. Responses will be described below.

व

Consider the motion of a particle in a plane as shown. For the locations shown, draw an arrow in the box to indicate the direction of the following vectors:
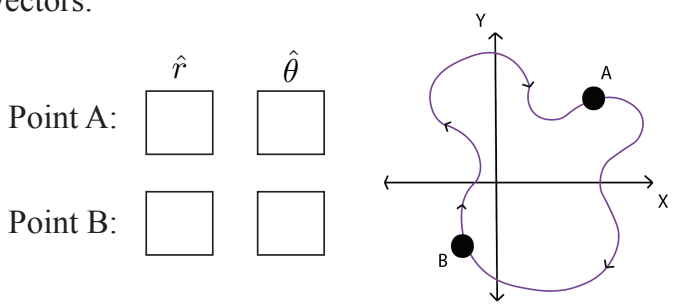

Figure 2. The revised unit vector question given to students in an interview type setting.

\section{Interviews:}

Analysis of the written responses suggested limitations of the data set. Student explanations on the written problems were typically brief and provided little insight into student thinking. In addition, our analysis suggested that we were unable to determine whether correct answers were based on correct reasoning. Some student explanations in support of correct answers appeared to be based on the motion of the object, which is irrelevant to the unit vectors. For that reason, we solicited student interview volunteers and revised the original question to limit this ambiguity. (see Figure 2)

The first revision was to the path of the particle. Rather than having it move counter clockwise (in the same direction as $\hat{\theta}$ ), the direction of motion was modified to go in a clockwise direction. Second, we made sure that $\hat{\theta}$ would not be coincidentally tangent to the path of the particle. As above, correct responses would have $\hat{r}$ away from the origin at each point and $\hat{\theta}$ perpendicular to $\hat{r}$ in a counter clockwise direction.

This revised question was posed in an interview setting to 4 students at CSUF who were taking a Math Methods course taught in a more traditional fashion by a non-PER professor. Students worked through the problem on a smart board as they spoke aloud their thought process. Student responses were transcribed and coded by members of the research team. Particular words and phrases were identified as being related and clusters of responses were developed through several iterations, described below in section IV.

\section{RESULTS AND DISCUSSION}

\section{Written Responses:}

Table 1 shows the number of students that answered the unit vector question on the written version correctly. While the success rate at CSUF is higher, the question was given after instruction and in class where the instructor made this topic a focus. Even with this focus, a third of students answered incorrectly, and some correct answers suggested incorrect underlying reasoning.

TABLE I. Percentage of students who obtained a correct answer for the written responses. $\mathrm{N}=$ number of students

\begin{tabular}{ccc}
\hline \hline School/Class & N Correct & Instruction \\
\hline NDSU: E \& M Theory & $5 \%(1)$ & Before \\
$(\mathrm{N}=21)$ & $64 \%(18)$ & After \\
$\begin{array}{c}\text { CSUF: Math Methods } \\
(\mathrm{N}=28)\end{array}$ & 6.07 \\
\hline \hline
\end{tabular}

Figure 3 shows the four common incorrect responses that students gave for either $\hat{r}$ or $\hat{\theta}$. These incorrect responses were arbitrarily labeled (1), (2), (3), (4).

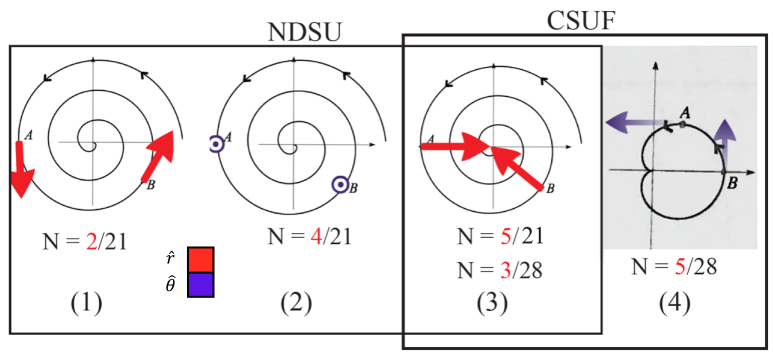

Figure 3. Common incorrect student responses for either $\hat{r}$ or $\hat{\theta}$ to the written version of the unit vector question. The red indicates $\hat{r}$ and the violet indicates $\hat{\theta}$.

Although explanations in the written data were often brief, we attempted to identify the underlying reasoning used by students. Many students drew vectors tangent to the path of the particle (answers (1) and (4)), apparently responding to the direction of motion. A few drew $\hat{r}$ tangent to the curve (answer (1)), which is clearly incorrect. However, several others drew $\hat{\theta}$ tangent to the curve (answer (4)), the correct $\hat{\theta}$ direction for point B but not for point $\mathrm{A}$ (with inconsistent answers for $\hat{r}$ ). This response also neglects the fact that polar coordinates have an orthogonal basis. At NDSU, several students indicated a direction out of the page (answer (2)). These students were likely either thinking of rotational motion and the angular velocity vector $\widehat{\omega}$ or considering spherical coordinates. In both groups, several students drew $\hat{r}$ pointing to the center (3), presumably based on forces or acceleration. However, written explanations were often incomplete. Due to the limited insight gained from the written responses, interviews were conducted to provide a deeper, in-the-moment probe of student thinking. 


\section{Interviews:}

The interviews were conducted late in the Math Methods course after all instruction on coordinate systems. However, all of the students who were interviewed struggled with this task in answering questions regarding coordinate systems and none generated a completely correct answer. These student responses gave us insight to the common incorrect responses seen in the written data, and others were seen for the first time. Figure 4 shows the responses given by each student, where the red arrows represent $\hat{r}$ and the violet arrows represent $\hat{\theta}$.

\section{a}

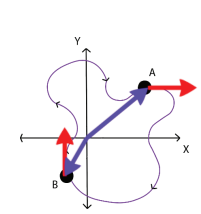

Student 1

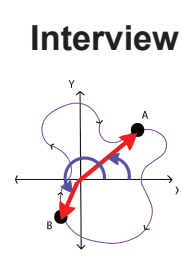

Student 2

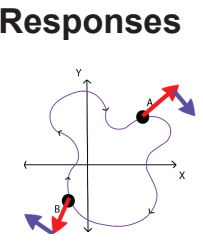

Student 3

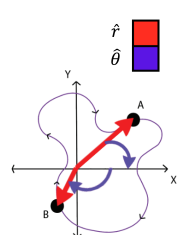

Student 4
Figure 4. Interview responses for for revised question.

As described above, student responses were coded and tentatively assigned to categories. For each student statement we identified key words that we then grouped into clusters of related terms. For example, 'goes' and 'travels' were identified with motion. Based on this analysis, we have tentatively identified a number of resources that students appear to be accessing in order to create their responses. In our model, to answer a physics question, a student will access a combination of resources (often unconsciously) and assemble them in a way that leads to an answer. Based on the structure of student responses, we classified clusters of related resources. It is important to note that these clusters reflect the researchers' identification; we do not mean to imply that students consider these ideas to be in clusters. (Indeed, different resources in a cluster might lead to contradictory responses.) Due to the small sample in this study, we also do not intend to claim that these represent all possible resources. For example, some of the written responses from NDSU show vectors pointing out of the paper, and responses of this type were not present in the interviews. Curvy arrows were not present in previous written responses, though they are present in previous research on 2D kinematics [10]. Below we describe each cluster and our tentative identification of resources in this cluster. Coordinate Cluster:

This cluster is based on the fundamental definitions of the coordinates in plane polar coordinates. Students returned to these definitions frequently in the interviews. We have identified two resources within this cluster. The first resource draws upon the basic definition of the $r$ coordinate: the distance from the origin to the point. The second similarly draws upon the definition of $\theta$ coordinate: the angle that is measured between the radial vector (the vector from the origin to the point of interest) and the positive $\mathrm{X}$-axis. None of the students in our sample used any other interpretation of either coordinate. While these resources draw upon the canonical definitions of $r$ and theta, students seemed to apply them in ways that were not productive for the unit vector task. Student 2 and 4 both drew $\hat{r}$ from the origin to each point. This suggested that the students had a clear understanding of the coordinate $r$ but were not able to activate other necessary resources, described below, needed to draw $\hat{r}$ correctly. (In particular, r-hat should be of unit length, whereas the two r-hat vectors here are drawn with different lengths.) Students 1, 2, and 4 explicitly referenced the definition of $\theta$ in different ways while drawing $\hat{\theta}$. For instance, student 1 correctly described the definition of theta: "theta is just the angle that is, this, it's, um, the angle that is at to the origin of this", gesturing that theta is measured counterclockwise starting from the positive $\mathrm{x}$-axis. However, he applied this definition in a way that was not productive, drawing $\hat{\theta}$ from the origin to each point since it allowed him to measure the correct value of $\theta$. Students 2 and 4 also stated the definition of theta but applied it in a completely different way, drawing not unit vectors but curved arcs with arrowheads.

\section{Motion Cluster:}

This cluster of resources was present in most of the interviews. We identified several resources in this cluster based on specific key words students used when drawing their vectors (for example: moving, traveling). The first resource is that when something is moving there is a vector tangent to the trajectory. Second, in motion there is a vector pointing towards the center or origin (We suspect that this requires motion perceived as 'circular' or 'rotation' but have not tested against other motions.) Finally, students seemed to use the sense of motion (e.g. clockwise or counter-clockwise, inward or outward) to determine their answers. Note that we have not specified which vector is tangent or radial, as student responses applied these ideas in a variety of ways that gave no two students the same answer. For instance, student 1 drew $\hat{r}$ rather than $\hat{\theta}$ tangent to the trajectory in the direction of motion. When asked to explain, student 1 responded, "I think, $\hat{r}$ goes this direction cause it's following the path and $r$ is just the direction of the vector moving". (This is parallel to answer type (1) from the written responses in Figure 3.) We interpreted this as the student relating $\hat{r}$ to the direction of motion, as if it were a velocity vector, but there is some ambiguity here. Student 3 , who we will discuss in more detail, seemed to activate several motion resources. Student 4 appeared to use the definition of theta from the coordinate cluster in conjunction with the direction of motion to determine the orientation of $\hat{\theta}$. Unit Vector Cluster:

In the unit vector cluster we have gathered resources that related to the canonical definitions of unit vectors and 
orthogonal coordinate systems. Unlike the other categories, we have included resources here that were not explicitly articulated by students, simply for a sense of completeness and to understand what kind of knowledge pieces we feel students might need to draw upon to have a complete understanding. The unit vector cluster consists of these pieces of knowledge: a unit vector is a vector; has 1-unit length; points in the direction of increasing coordinate; and is dimensionless. While we might have assumed students would have ready access to these resources, responses suggest we cannot take them for granted. The responses given by students 2 and 4 for $\hat{\theta}$ and $\hat{r}$ suggest that they either did not have the knowledge or were not able to activate fundamental resources that a unit vector cannot be curved and has a magnitude of 1 .

Student 3 did productively use the resource that $\hat{r}$ and $\hat{\theta}$ should be perpendicular and stated, " $\hat{r}$ is always away from the origin or whatever center is and $\hat{\theta}$ is always perpendicular to that either in clockwise or counter clockwise", though he chose the wrong direction.

\section{Location Dependent Resource:}

This resource is on a different logical footing than the others, as it is basis-specific. In polar and spherical coordinates, the unit vectors change with location, while in Cartesian coordinates unit vectors are independent of location. We asked a follow-up question regarding the unit vectors in Cartesian coordinates. For the same two points in figure 2 , we asked students to draw $\hat{\imath}$ and $\hat{\jmath}$. While most students did not find this difficult, student 1 drew the Cartesian unit vectors differently for each point: "We're looking at the individual points. So they have, each point has their own set of direction". Student 4 originally drew the Cartesian unit vectors differently for each point as well and stated, "they would be pointing in the opposite direction because it's in the opposite quadrant". After additional thought, student 4 realized something was not right and changed their answer to point B to be consistent with point A.

Special comment on Student 3:

The responses of student 3 were of particular interest. Student 3 seemed to invoke all the necessary resources needed to answer the question correctly. As seen in Figure 4 , the student's answers for $\hat{r}$ and $\hat{\theta}$ were almost correct. However, the student's responses proved to more fluid than one might expect. The student started by drawing $\hat{r}$ correctly away from the origin for each point. The student stated that polar coordinates is an orthogonal basis and clearly pointed out that there are two ways that $\hat{\theta}$ can be oriented and still be orthogonal to $\hat{r}$. When trying to reason through which orientation was more correct, the student began to second guess the answer to $\hat{r}$. They decided that $\hat{r}$ should instead point towards the origin, and erased and redrew this vector. After redrawing $\hat{r}$, the student asked, "Are we going from position or motion?", suggesting the student was conflicted in whether to apply the position resource or the motion resource. We believe that the position resource suggested that $\hat{r}$ should point outwards, while the motion resource suggested instead that it should be pointing towards the center due to the inward path of the particle at point B. In the end the student chose the correct $\hat{r}$ direction, again erasing and redrawing. However, even after settling on the correct $\hat{r}$, when asked to justify the [incorrect] choice of orientation for $\hat{\theta}$, the student said, "I was actually going for motion for that". We interpret this as a student activating an additional, context-dependent resource due to the motion,

\section{CONCLUSION}

Examining the student interviews gave us much needed insight into students' written responses. While not one student was able to give a complete correct answer, a variety of productive and unproductive resources were identified. Although further research is needed, some preliminary conclusions are possible. First, despite incorrect answers that might be described as difficulties, students were knowledgeable and activated productive resources when answering physics question pertaining to polar coordinates. However, students also activated additional resources that influence their answer in unproductive ways. Second, students appeared to lack some fundamental knowledge regarding what a unit vector is. Not having access to these fundamental resources made it difficult for students to check answers. All of these questions are in the context of motion but we plan future work involving other contexts to examine the extent to which resources are context dependent.

\section{REFERENCES}

[1] D. Hammer. Am. J. Phys. 68, S52-S59 (2000).

[2] M. E. Loverude and B. S. Ambrose, Phys. Rev. STPER, 020002 (2015).

[3] B. Wilcox et al., Phys. Rev. ST-PER, 020119 (2013)

[4] B. E. Hinrichs, B. E. (2010, October). PERC Proc.

[5] E. C. Sayre and M.C. Wittmann, Phys. Rev. ST-PER, 020105 (2008).

[6] T. Paoletti, K.C. Moore, J. Gammaro, and S. Musgrave, Proc. of the XVI Conf. on RUME (2013). [7] Montiel et al. Educ. Stud. in Mathematics, 72(2), 139160. (2009).

[8] P. Heron, PROCEEDINGS-INTERNATIONAL SCHOOL OF PHYSICS ENRICO FERMI. Vol. 156. IOS Press; Ohmsha; 1999, 2004

[9] B. Farlow and W. Christensen, submitted to 2016 PERC Proceedings.

[10] R. P Springuel, M. C. Wittmann, and J. R. Thomspon, Phys Rev ST-PER 3, 020107 (2007). 